\title{
THE STABILITY OF SMOOTHING BY LEAST SQUARES
}

\author{
HERBERT S. WILF ${ }^{1}$
}

1. Introduction. Let $2 n+1$ numbers $u_{-n}, \cdots, u_{0}, \cdots, u_{n}$ be given, and let $P(x)$ denote the polynomial of degree $2 k$ which best approximates the points $\left(\left(-n, u_{-n}\right), \cdots,\left(n, u_{n}\right)\right)$ in the sense

$$
\sum_{l=-n}^{n}\left\{P(l)-u_{l}\right\}^{2}=\text { minimum }
$$

of least squares. Then

$$
P(0)=\sum_{l=-n}^{n} \rho_{l} u_{l}
$$

where the weights $\rho_{l}$ depend on $n$ and $k$ but not on the $u_{l}$. The weights being so determined, let $\left\{u_{l}\right\}_{-\infty}^{\infty}$ be a given sequence and consider the smoothing formula

$$
u_{r}^{\prime}=\sum_{l=-n}^{n} \rho_{l} u_{r-l} \quad(r=\cdots,-1,0,1, \cdots) .
$$

The question of stability arises when one asks for the behavior of the high-order iterates of the smoothing process (3). Roughly speaking, (3) is stable if the $m$-times smoothed sequence approaches an asymptotically smooth shape independent of the initial sequence, and unstable otherwise. The question of stability has been investigated by DeForest [1], Schoenberg [2], [3], [4], and others, with the result that if the process (3) is stable then

$$
|h(\theta)|=\left|\sum_{l=-n}^{n} \rho_{l} \cos l \theta\right|<1 \quad(0<\theta<2 \pi) .
$$

We shall refer to the above as the discrete smoothing problem.

The continuous analogue is obvious. If $f(x)$ is given on $(-1,1)$, and $P(x)$ is the best approximation to $f(x)$ in the least squares sense, then

$$
P(0)=\int_{-1}^{1} \rho(t) f(t) d t
$$

Received by the editors July 3, 1963.

1 This work was supported by the National Science Foundation. 
and stability requires that

$$
|h(\theta)|=\left|\int_{-1}^{1} \rho(t) \cos t \theta d t\right|<1 \quad(\theta \neq 0) .
$$

The criteria (4), (6) would obviously be satisfied if the weights were nonnegative, but this is far from the case in least squares smoothing. In this note we show that smoothing by least squares is stable in the continuous case, the discrete case remaining open. We note further the intimate connection between these problems and the investigations of R. G. Cooke [5] on the Gibbs phenomenon in FourierBessel series.

2. A preliminary result. Let $J_{\nu}(x)$ denote the usual Bessel function of the first kind, and let $j_{p p}$ be its $p$ th positive zero. It is known that [6]

$$
j_{\nu 1}=\nu+A \nu^{1 / 3}+O(1) \quad(\nu \rightarrow \infty),
$$

where $A=1.855757 \cdots$ is the root of a certain transcendental equation. In this section we wish to prove that

(8)

$$
\begin{gathered}
\lim _{\nu \rightarrow \infty} \frac{\int_{0}^{j_{\nu 1}} \frac{J_{\nu}(t)}{\sqrt{ }(t)} d t}{\int_{0}^{\infty} \frac{J_{\nu}(t)}{\sqrt{ }(t)} d t} \\
=\frac{1}{\sqrt{ }(6)}+\sqrt{ }\left(\frac{2}{3 \pi}\right) \int_{0}^{(2 \Lambda)^{3 / 2} / 3} \frac{\cos \left(t-\frac{\pi}{4}\right)}{\sqrt{ }(t)} d t .
\end{gathered}
$$

To do this we shall need the asymptotic formulas of Rayleigh [6, p. 234],

(9) $J_{\nu}(\nu \sec \beta)=\left\{\frac{\nu \pi \tan \beta}{2}\right\}^{-1 / 2} \cos \left\{\nu(\tan \beta-\beta)-\frac{1}{4} \pi\right\}+O\left(\nu^{-3 / 2}\right)$, and of Debye [6, p. 243],

$$
\begin{aligned}
J_{\nu}(\nu \operatorname{sech} \beta)=\{2 \pi \nu \tanh \beta\}^{-1 / 2} \exp \{\nu(\tanh \beta-\beta)\}(1+ & o(1)) \\
& (\nu \rightarrow \infty) .
\end{aligned}
$$

Now we have 
$1964]$

$$
\begin{aligned}
\int_{0}^{\nu} \frac{J_{\nu}(t)}{\sqrt{ }(t)} d t & =\sqrt{ }(\nu) \int_{0}^{\infty} J_{\nu}(\nu \operatorname{sech} \beta) \frac{\sinh \beta}{(\cosh \beta)^{3 / 2}} d \beta \\
& \sim \frac{1}{\sqrt{ }(2 \pi)} \int_{0}^{\infty} \frac{(\sinh \beta)^{1 / 2}}{\cosh \beta} \exp \nu(\tanh \beta-\beta) d \beta \\
& \sim \frac{1}{\sqrt{ }(2 \pi)} \int_{0}^{\infty} \sqrt{ }(\beta) e^{-\nu \beta^{2} / 3} d \beta \quad(\nu \rightarrow \infty) \\
& =\frac{1}{\sqrt{ }(6 \nu)} .
\end{aligned}
$$

Further

$$
\begin{aligned}
\int_{\nu}^{j_{1} \nu} & \frac{J_{\nu}(t)}{\sqrt{ }(t)} d t \\
= & \sqrt{ }(\nu) \int_{0}^{\sec ^{-1}\left(j_{\nu} / \nu\right)} J_{\nu}(\nu \sec \beta) \frac{\sin \beta}{(\cos \beta)^{3 / 2}} d \beta \\
= & \sqrt{ }\left(\frac{2}{\pi}\right) \int_{0}^{\sec ^{-1}\left(j_{1} / \nu\right)} \cos \left\{\nu(\tan \beta-\beta)-\frac{\pi}{4}\right\} \frac{(\sin \beta)^{1 / 2}}{\cos \beta} d \beta \\
& +O\left(\nu^{-1}\right) \int_{0}^{\sec ^{-1}\left(j_{1 \nu} / \nu\right)} \sqrt{ }(\beta) \cos \left\{\frac{2}{3}-\frac{\pi}{4}\right\} d \beta \quad(\nu \rightarrow \infty) \\
\sim & \sqrt{ }\left(\frac{2}{3 \pi \nu}\right) \int_{0}^{(\nu / 3)\left(\sec ^{-1}\left(j_{1} / \nu\right)\right)^{3}} t^{-1 / 2} \cos \left(t-\frac{\pi}{4}\right) d t \\
\sim & \sqrt{\left(\frac{2}{3 \pi \nu}\right) \int_{0}^{(2 \Lambda)^{3 / 2} / 3} \frac{\cos \left(t-\frac{\pi}{4}\right)}{\sqrt{ }(t)} d t .}
\end{aligned}
$$

Adding these results,

$$
\int_{0}^{j_{1} \nu} \frac{J_{\nu}(t)}{\sqrt{ }(t)} d t \sim C \nu^{-1 / 2} \quad(\nu \rightarrow \infty)
$$

where $C$ is the constant on the right of (8). But it is well known that

$$
\int_{0}^{\infty} \frac{J_{\nu}(t)}{\sqrt{ }(t)}=\frac{1}{\sqrt{ }(2)} \frac{\Gamma\left(\frac{\nu}{2}+\frac{1}{4}\right)}{\Gamma\left(\frac{\nu}{2}+\frac{3}{4}\right)} \sim \frac{1}{\sqrt{ }(\nu)} \quad(\nu \rightarrow \infty),
$$


and the assertion (8) is proved.

3. The stability of the continuous smoothing process. Let $f(t)$ be given on $(-1,1)$, and let $P(t)$ denote the best least squares approximation to $f(t)$ by a polynomial of degree $2 k$. Then

$$
P(t)=\sum_{\nu=0}^{2 k} P_{\nu}(t) \int_{-1}^{1}\left(\nu+\frac{1}{2}\right) P_{\nu}(\tau) f(\tau) d \tau
$$

where $P_{r}(t)$ is the usual Legendre polynomial. Hence

$$
\begin{aligned}
P(0) & =\int_{-1}^{1} f(t)\left\{\sum_{\nu=0}^{2 k}\left(\nu+\frac{1}{2}\right) P_{\nu}(0) P_{\nu}(t)\right\} d t \\
& =\left(k+\frac{1}{2}\right) P_{2 k}(0) \int_{-1}^{1} f(t) \frac{P_{2 k+1}(t)}{t} d t .
\end{aligned}
$$

Thus the weight function $\rho(t)$ in (5) is

$$
\rho(t)=\left(k+\frac{1}{2}\right) P_{2 k}(0) \frac{P_{2 k+1}(t)}{t},
$$

and to prove stability we must examine the function

$$
h(\theta)=\left(k+\frac{1}{2}\right) P_{2 k}(0) \int_{-1}^{1} \frac{P_{2 k+1}(t)}{t} \cos t \theta d t .
$$

But

$$
\begin{aligned}
h^{\prime}(\theta) & =-\left(k+\frac{1}{2}\right) P_{2 k}(0) \int_{-1}^{1} P_{2 k+1}(t) \sin t \theta d t \\
& =-\left(k+\frac{1}{2}\right) P_{2 k}(0) 2^{-2 k-1}(2 k+1) !^{-1} \times \int_{-1}^{1} \sin t \theta \frac{d^{2 k+1}}{d t^{2 k+1}}\left(t^{2}-1\right)^{2 k+1} d t \\
& =\left\{(-1)^{k}\left(k+\frac{1}{2}\right) \frac{P_{2 k}(0)}{2^{2 k+1}(2 k+1) !}\right\} \theta^{2 k+1} \times \int_{-1}^{1} \cos t \theta\left(t^{2}-1\right)^{2 k+1} d t \\
& =-\sqrt{ }\left(\frac{\pi}{2}\right)(2 k+1) 4^{-k}\left(\begin{array}{c}
2 k \\
k
\end{array}\right) \frac{J_{2 k+3 / 2}(\theta)}{\sqrt{ }(\theta)},
\end{aligned}
$$

and so

$$
h(\theta)=1-\left\{\sqrt{ }\left(\frac{\pi}{2}\right)(2 k+1) 4^{-k}\left(\begin{array}{c}
2 k \\
k
\end{array}\right)\right\} \int_{0}^{\theta} \frac{J_{2 k+3 / 2}\left(\theta^{\prime}\right)}{\sqrt{ }\left(\theta^{\prime}\right)} d \theta^{\prime}
$$

or, finally, 


$$
h(\theta)=1-\frac{\int_{0}^{\theta} t^{-1 / 2} J_{2 k+3 / 2}(t) d t}{\int_{0}^{\infty} t^{-1 / 2} J_{2 k+3 / 2}(t) d t} .
$$

Now we must show that

$$
-1<h(\theta)<1 \quad(\theta \neq 0) .
$$

For the right-hand inequality we must show that the numerator in (13) is positive for $\theta \neq 0$. But Cooke [5] actually proved that the numbers

$$
\left|\int_{j_{\nu}}^{j_{\nu}, p+1} t^{-1 / 2} J_{\nu}(t) d t\right| \quad(p=1,2, \cdots)
$$

form a decreasing sequence, where $j_{v p}$ is previously defined, which clearly implies the desired result.

For the left inequality in (14) we must show that the ratio of integrals in (13) is less than 2. By the result of Cooke just quoted, the maximum of this ratio is at $\theta=j_{2 k+3 / 2,1}$, and since the constant in (8) has the value $1.502 \cdots<2$ the result is established for all large enough $k$, and we have proved Theorem 1. There is an integer $k_{0}$ such that least squares smoothing which preserves polynomials of degree $k \geqq k_{0}$ satisfies the stability condition (6).

Presumably $k_{0}=0$, but we cannot prove this.

\section{REFERENCES}

1. H. H. Wolfenden, On the development of formulae for graduation by linear compounding, with special reference to the work of Erastus L. DeForest, Trans. Amer. Math. Soc. 26 (1925), 81-121.

2. I. J. Schoenberg, On smoothing operations and their generating functions, Bull. Amer. Math. Soc. 59 (1953), 199-230.

3. - Contributions to the problem of approximation of equidistant data by analytic functions. Part A, Quart. Appl. Math. 4 (1946), 45-99; Contributions to the problem of approximation of equidistant data by analytic functions. Part B, ibid. 4 (1946), $112-141$.

4. - Some analytical aspects of the problem of smoothing. Studies and essays presented to $R$. Courant on his 60th birthday, pp. 152-156, Interscience, New York, 1948.

5. R. G. Cooke, Gibbs' phenomenon in Fourier-Bessel series and integrals, Proc. London Math. Soc. 27 (1928), 171-192.

6. G. N. Watson, A treatise on the theory of Bessel functions, Cambridge Univ. Press, Cambridge, 1950.

University of Pennsylvania 